\title{
MODELLING OF A DIESEL ENGINE COMMON RAIL INJECTION SYSTEM
}

\author{
Christophe Gauthier ${ }^{* * * *}$, Olivier Sename ${ }^{* *}$ \\ Luc Dugard $^{* *}$, Guillaume Meissonnier* \\ * Delphi Diesel System, 9 Boulevard de l'industrie \\ 41000 Blois Cedex, FRANCE \\ \{christophe.l.gauthier;guillaume.meissonnier\}@delphi.com \\ ** Laboratoire d'Automatique de Grenoble, ENSIEG-BP 46, \\ 38402 Saint Martin d'Hères Cedex, FRANCE \\ \{olivier.sename;luc.dugard\}@inpg.fr
}

\begin{abstract}
This paper is devoted to the modelling of a Diesel Common Rail Injection System. The objective is twofold : to build a simulator which will be used later for the rail pressure control scheme validation, and to get linearized models for control design. A mathematical model, based on physical equations is developed and used as a simulator. Then time responses of the rail pressure of a real engine and of the simulator are compared in order to check the accuracy of the model. A frequency domain approach is finally proposed to validate linearized control-oriented models. Copyright (C) 2005 IFAC
\end{abstract}

Keywords: Diesel engine, Common Rail system, rail pressure control

\section{INTRODUCTION}

Automotive systems are becoming more and more complex and engine control (gasoline, diesel or common rail) now constitutes a large part of it (EGR (Rucker et al., n.d.), idle, turbocharger, cruise control, etc...) in which automatic control has a real place (Kiencke and Nielsen, 2000; Gissinger and Le Fort-Piat, 2002; van Nieuwstadt et al., 1999). In particular Common Rail systems have been developed to reduce noise, exhaust emissions and fuel consumption and at the same time to increase performances.

The working principle is to inject a precise quantity of fuel at high pressure (Guerrassi and Dupraz, 1998; Guerrassi et al., 2002). The pressure demand is mapped against several parameters, mainly engine speed and torque demand. This pressure must be able to vary typically from 230 bar up to 1600 bar within a tolerance of $1 \%$ and steep gradients (e.g. up to $\pm 3000 \mathrm{bar} / \mathrm{s}$ ).
Five main components compose this system as shown in figure 1 . The first one is the rail which is a pressurized tank feeding the injectors. Then a high pressure (HP) pump, driven by the engine, fills the rail and increases its pressure. The higher the engine speed is, the greater the pump flow is. In order to control the pump flow, an inlet metering valve (IMV) is placed at the HP pump inlet. Then injectors inject fuel from the rail into the cylinders, which means that this flow cannot be used for rail pressure control. The last component, the high pressure valve (HPV), allows to control the output flow of the rail.

The industrial performances presented above are reached with components from a new technology. Nevertheless, if rail pressure is poorly controlled, system performances may be degraded (e.g. increase of combustion noise or of tailpipe emissions). This stresses the importance of having an 


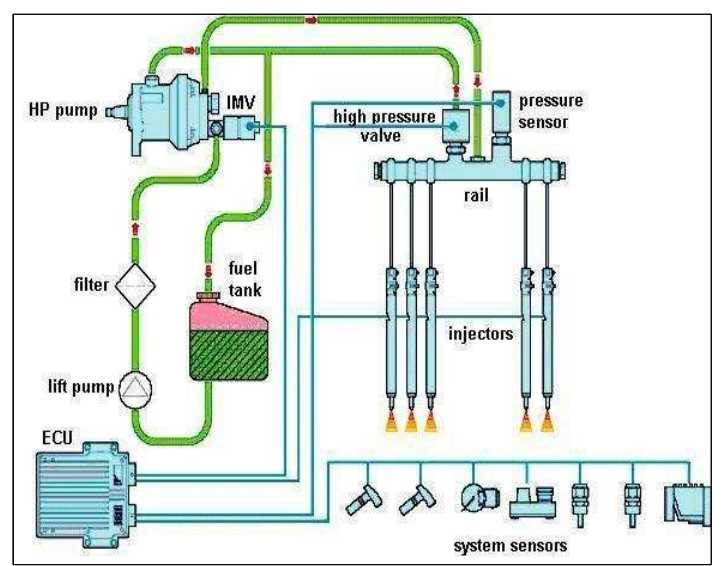

Fig. 1. Common rail system

efficient rail pressure control. Today, the different kinds of systems that are running differ in their components. For example, depending on the engine, the capacity of the HP pump and the rail volume can be different. This means that the structure of the system is fixed but allows different dynamics. These dynamics are non-linear and depend on parameters such as fuel temperature, rail pressure, etc... For example, the output pump flow would not have the same effect on the rail pressure if the pressure is low or high. Presently, the method used for controller design consists of building maps which define each operating point where system dynamics are different. Then, at each operating point, an identification of the system model is performed. Finally, controllers are optimized for all models. These tasks are long as there are many breakpoints in the maps. The motivations behind the proposed methodology are twofold. The first one is to have a mathematical model with calibration parameters as close as possible to the real system so that it can be possible to predict dynamics for each system, i.e. with different HP pumps or rail volumes. The objective is then to know the effect of parameters on the system and therefore to minimize the control design process time. The second motivation is to improve rail pressure control. The current control is composed of two independent PID controllers, each one driving one actuator, for one unique output, and the control strategy has three modes: IMV only in control, HPV only in control and both IMV and HPV in control. Each SISO (single input single output) case (i.e. IMV or HPV only in control) is rather satisfactory but the MISO (multi-inputs single output) one (i.e. IMV and HPV in control) often leads to unsteady closedloop behaviors. A first step to a good control design is the modelling of the rail pressure system (section 2). The model validation in time and frequency domains are presented in sections 3 and 4 .

\section{MODELLING}

The figure 2 shows the synoptic of the rail pressure system. The only measurement is the rail pressure $P_{r}$ (the flows $Q_{p m p}, Q_{i n j}$ and $Q_{h p v}$ are not measured). $V_{i m v}, X_{i m v}$ and $V_{h p v}$ are presented below.

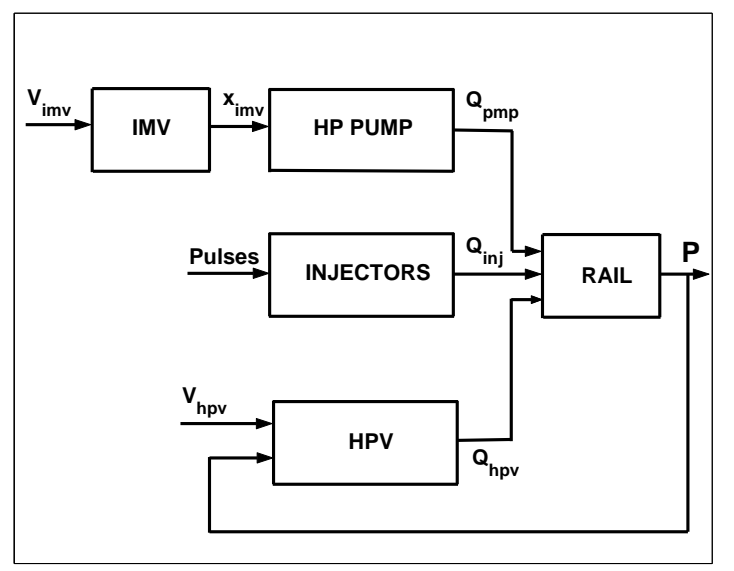

Fig. 2. synoptic of the system

Pulses are the current impulses for each injector; their length is determined by the fuel demand and the rail pressure.

\subsection{Fuel tank : the rail}

The rail is a pressurized fuel tank feeding the injectors. Two kinds of flows cross the rail : the control flow which allows to keep the right pressure, and the running flow used by injectors. The rail subsystem satisfies the following dynamical equation :

$$
\dot{P}_{r}=\frac{K\left(P_{r}, T\right)}{V} \cdot\left(Q_{p m p}-Q_{i n j}-Q_{h p v}\right)
$$

where $P_{r}$ is the rail pressure $(P a), T$ is the fuel temperature $\left({ }^{\circ} \mathrm{C}\right), \mathrm{K}$ is the bulk modulus $(\mathrm{Pa})$, $V$ is the rail and pipes volume $\left(m^{3}\right), Q_{p m p}, Q_{i n j}$ and $Q_{h p v}$ are respectively the H.P. pump flow, the injectors flow and the HPV flow $\left(\mathrm{m}^{3} / \mathrm{s}\right)$. The bulk modulus is a function of the fuel temperature and the pressure, given as follows :

$$
K\left(P_{r}, T\right)=\mathcal{F}_{t}(T) \cdot \tanh \left(\frac{P_{r}}{a}\right)+\mathcal{F}_{p}\left(P_{r}\right)
$$

where $\mathcal{F}_{t}(T)$ is a function of the temperature, $\mathcal{F}_{p}\left(P_{r}\right)$ is a function of the rail pressure and $a$ is a coefficient.

\subsection{Filling: the Inlet Metering Valve and the H.P. pump}

The Inlet Metering Valve (IMV) is a spring-mass system, moved by an electromagnetic field coming from a coil crossed by a current $I_{i m v}$. The mass uncovers an area which controls the H.P. pump 


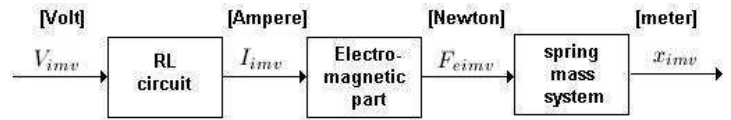

Fig. 3. Synoptic of the IMV

filling. Figure 3 is the IMV synoptic showing the different subsystems. Equations from this synoptic are as follows ( $s$ denoting the Laplace variable) : The current $I_{i m v}$ is :

$$
I_{i m v}(s)=\frac{\frac{1}{R_{i m v}}}{1+\frac{L_{i m v}}{R_{i m v}} \cdot s} \cdot V_{i m v}(s)
$$

with $I_{i m v}$ the current through the coil $[A], V_{i m v}$ the voltage applied to the coil $[V], R_{i m v}$ the resistance of the coil $[\Omega]$ and $L_{i m v}$ the inductance of the coil $[H]$. The electromagnetic force $[N]$ $F_{\text {eimv }}$ i :

$$
F_{\text {eimv }}(s)=K_{\text {eimv }} \cdot I_{i m v}(s)
$$

where $K_{\text {eim }}$ is a coefficient [N. $A^{-1}$ ], which can be considered as a constant(due to the IMV design). Finally the spring mass system $x_{i m v}$ is :

$$
x_{i m v}(s)=\frac{\frac{1}{K_{i m v}}}{1+\frac{\nu_{i m v}}{K_{i m v}} \cdot s+\frac{m_{i m v}}{K_{i m v}} \cdot s^{2}} \cdot F_{\text {eimv }}(s)
$$

with $x_{i m v}$ the position of the mass $[m], K_{i m v}$ the sum of the two springs stiffness $\left[N . m^{-1}\right], m_{i m v}$ the moving mass $[k g]$ and $\nu_{i m v}$ the viscosity of the fuel $[\mathrm{N} / \mathrm{m} / \mathrm{s}]$ The IMV position modifies the flow area for the fuel. The Bernoulli's simplified flow equation is :

$$
Q_{i m v}=C_{q i m v} \cdot S_{i m v} \cdot \sqrt{\frac{2 \cdot \Delta P_{i}}{\rho}}
$$

with $C_{q i m v}$ the flow coefficient, $S_{i m v}$ the IMV flow area $\left[m^{2}\right], \Delta P_{i}$ the absolute value of pressure difference on each side of the IMV $[P a]$ and $\rho$ the fuel density $\left[\mathrm{kg} / \mathrm{m}^{3}\right]$. The coefficient $C_{q i m v}$ is a function of the IMV position $x_{i m v}$ and of the absolute pressure difference $\Delta P$, given as :

$$
C_{q i m v}=C_{q m a x} \cdot \tanh \left(\frac{2 \cdot \frac{h_{d}\left(x_{i m v}\right)}{\nu_{i m v}} \cdot \sqrt{\frac{2 \cdot \Delta P_{i}}{\rho}}}{l a m c}\right)
$$

with $C_{q \max }$ the maximum flow coefficient, $h_{d}$ the hydraulic diameter $\left[m^{2}\right], \rho$ the fuel density $\left[\mathrm{kg} / \mathrm{m}^{3}\right]$ and $l a m c$ the critical flow number. The HP pump flow equation is the IMV flow equation (6) filtered by the pump dynamics.

$$
Q_{p m p}=G_{p m p}(s) \cdot Q_{i m v}
$$

with $Q_{p m p}$ the output pump flow $\left[\mathrm{m}^{3} \cdot \mathrm{s}^{-1}\right]$ and $G_{p m p}(s)$ the dynamics of the HP pump.

\subsection{Discharge : the High Pressure Valve}

The HPV flow is controlled by the position of a ball on a conical seat (IMAGINE, 2003). This ball uncovers a flow area. Both the mass-spring system and the electromagnetic force, from an electrical source, push the ball on its seat. On the other hand, the rail pressure acts on the ball and tends to open the orifice. Figure 4 shows a simple scheme of its principle where the HPV flow equation is :

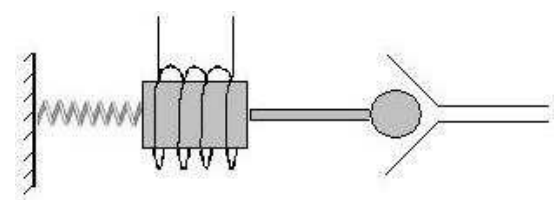

Fig. 4. HPV

$$
Q_{h p v}=C_{q h p v} \cdot S_{h p v} \cdot \sqrt{\frac{2 \cdot \Delta P_{h}}{\rho}}
$$

with $C_{q h p v}$ the flow coefficient, $S_{h p v}$ the flow area $\left[m^{2}\right], \Delta P_{h}$ the unsigned difference between rail pressure and tank pressure $[\mathrm{Pa}]$ and $\rho$ the fuel density $\left[\mathrm{kg} / \mathrm{m}^{3}\right]$. The flow coefficient $C_{q h p v}$ in this case is constant and is equal to the maximum flow coefficient because the value of $\Delta P_{h}$ is high and the HPV flow can always be considered as turbulent and never as laminar. The flow area $S_{h p v}$ depends on the ball position $x_{h p v}$ as following :

$$
S_{h p v}=\pi \cdot \sin \left(\frac{\pi}{2}-\theta\right) \cdot \cos \left(\frac{\pi}{2}-\theta\right) \cdot d_{b} \cdot x_{h p v}
$$

with $\theta$ the semi-angle of the conical seat $[\mathrm{rad}], d_{b}$ is the ball diameter $[m]$ and $x_{h p v}$ is the ball position $[m]$. The spring-mass system can be described by :

$$
m \ddot{x}_{h p v}=F_{h}-F_{r}-F_{e h p v}-F_{j e t}-F_{\nu_{h p v}}
$$

where $m$ is the mass of the moving parts $[\mathrm{kg}], F_{h}$ is the hydraulic force, $F_{r}$ is the spring force, $F_{\text {ehpv }}$ is the electromagnetic force, $F_{j e t}$ is the jet force and $F_{\nu_{h p v}}$ is the friction force. All forces are in Newton.

Similarly to the IMV, the electromagnetic force $F_{\text {ehpv }}$ is as follows :

$$
F_{e h p v}(s)=K_{e h p v} \cdot \frac{\frac{1}{R_{h p v}}}{1+\frac{L_{h p v}}{R_{h p v}} \cdot s} \cdot V_{h p v}(s)
$$

with $K_{\text {ehpv }}$ the electromagnetic coefficient [N.A $A^{-1}$, $R_{h p v}$ the resistance of HPV's coil $[\Omega], L_{h p v}$ the inductance of HPV's coil $[H]$ and $V_{h p v}$ the voltage applied to the HPV's coil [V]. $F_{h}$ is due to the rail pressure which acts on the active area of the ball and tends to open the orifice.

$$
F_{h}=S_{a} \cdot P_{r}
$$

with $S_{a}$ the active area $[m]$ and $P_{r}$ the rail pressure $\left[P_{a}\right] . F_{j e t}$ is an hydraulic force which tends to close the orifice :

$$
F_{j e t}=2 \cdot C_{q h p v} \cdot S_{h p v} \cos (\theta) \cdot \Delta P_{h}
$$


The HPV is therefore a system with two inputs which are $V_{h p v}$ and $P_{r}$ and one output $Q_{h p v}$.

\subsection{Injectors}

The design of the injector leads to three kinds of flow : the injected flow, the control flow and the leakage flow :

$$
Q_{i n j}=\mathcal{F}_{i n j}(F)+\mathcal{F}_{c t r l}\left(P_{r}, F\right)+\mathcal{F}_{l k g}\left(P_{r}\right)
$$

with $\mathcal{F}_{i n j}(F)$ the injected flow $\left[m^{3} . s^{-1}\right], F$ the injection demand $[\mathrm{mg} /$ stroke $], \mathcal{F}_{c t r l}\left(P_{r}, F\right)$ the control flow $\left[m^{3} . s^{-1}\right]$ and $\mathcal{F}_{l k g}\left(P_{r}\right)$ the leakage flow $\left[m^{3} \cdot s^{-1}\right]$.

To conclude, let us mention that all the parameters above used are known except $K_{\text {ehpv }}$, which needs to be adjusted by some ad hoc methodology, as we will see later.

\section{TIME DOMAIN VALIDATION OF THE NON LINEAR MODEL}

Three validations are made in this section. The first one is to ensure that simulator behaves as the true system when operating in open loop. The two others are the rail-HPV subsystem validation (considering that both the HP pump and the injectors have a constant flow (see figure 2)) and the HP pump model validation. Notice that it is not necessary to validate the IMV model because it is a simple spring mass system with known parameters.

\subsection{Validation of the simulator behavior}

A simple simulation (Fig. 5) can provide information on the shape of each flow and on the rail pressure. The engine speed is fixed at 1000 revolutions per minute $([\mathrm{rpm}])$, the IMV is fully opened (no current), the HPV has a constant current, the fuel demand is $21.6[\mathrm{mg} /$ stroke $]$ and the temperature of the fuel is $40^{\circ} \mathrm{C}$. The behavior of the rail pressure corresponds to a cyclo-stationary point with a constant mean value. On figure 5, the second plot is the HP pump flow, the third plot is the injected flow and the last plot is the HPV flow. The input flow (HP pump) tends to increase the rail pressure and the outputs flows (HPV and injectors) tend to decrease it, as one can see on the first plot. This first test emphasizes the relevance of the model, in particular w.r.t the shapes of the three output flows $Q_{p m p}, Q_{i n j}$ and $Q_{h p v}$ which are satisfactory (this cannot be checked in practice as the flows are not measured).

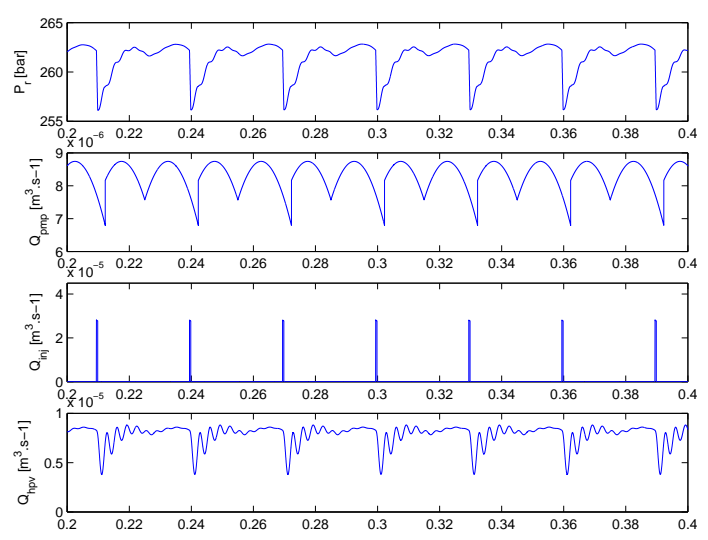

Fig. 5. Instantaneous rail pressure and flows system

\subsection{Validation of the rail-HPV subsystem}

The rail-HPV model and the real plant output are compared (Fig. 6). The HPV actuator is stimulated by a PRBS signal while the IMV input is constant. This time domain validation shows if our model is to close to the real system on a large frequency range. Note that the value of $K_{\text {ehpv }}$ in the model has been tuned to fit the real measurement, as described with more details in the next section. This points out that the dynamic

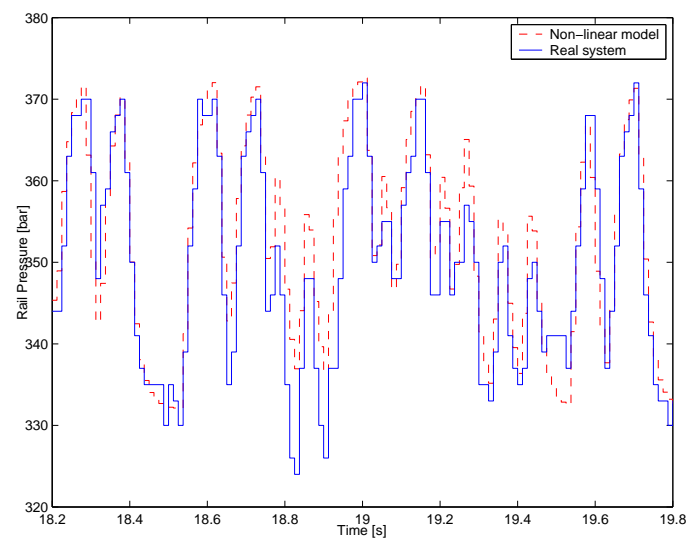

Fig. 6. Simulation and real system time responses behavior of the model is accurate, when compared with real data.

\subsection{Validation of the HP pump subsystem}

The model of the HP pump cannot be compared with real data as the output pump flow $Q_{p m p}$ cannot be measured. A simulation software (AmeSim) is used to obtain a realistic and fine model of the HP pump. Figure 7 shows both the output pump flow and the pump volume excited by a PRBS on the inlet of the HP pump $\left(x_{i m v}\right)$. The output pump volume is the output pump flow integrated between two injections (see Fig 5) providing a best comparaison. The non-linear model of the HP pump provides a good time response compared to the AmeSim model. 

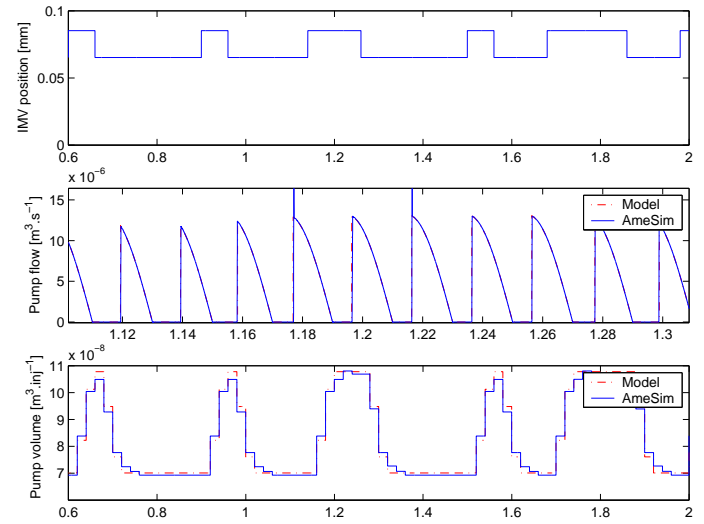

Fig. 7. Time response of the HP pump

\section{VALIDATION IN THE FREQUENCY DOMAIN OF THE LINEARIZED MODEL}

This section concerns the model validation in the frequency domain. The analysis is performed on the tangent linearized model of the previous non-linear model. The frequency response of the real plant is here identified and compared with the Bode diagram of the linearized model. For this purpose, a spectral estimation method (Chebassier, 2001; Ljung, 2001) is used instead of usual parametric identification method (e.g ARMAX, etc...). This method is briefly described below. $G$ is the model of the plant and $H$ is the model of the noise.

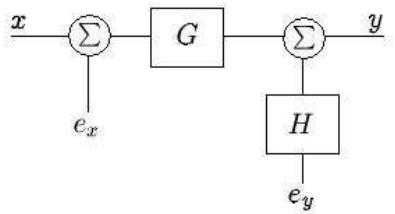

Fig. 8. Process model representation

$$
S_{y}=|G|^{2} \cdot S_{x}+|G|^{2} \cdot S_{e x}+|H|^{2} \cdot S_{e y}
$$

where $S_{y}, S_{x}, S_{e x}$ and $S_{e y}$ are respectively the spectral estimation of $y, x, e_{x}$ and $e_{y}$. Assuming that $e_{x}$ and $e_{y}$ are some noises with small magnitude, an estimation of the frequency response of the system is :

$$
|G(\omega)|=10 \cdot \log \left(\frac{S_{y}(\omega)}{S_{x}(\omega)}\right)
$$

To ensure that the assumption - $e_{x}$ and $e_{y}$ are noises - is true for all $\omega$, the coherence function is calculated. The result range is from 0 to 1 . If the noises are small compared to $x$, the result is close to 1 and vice versa.

$$
\Gamma^{2}(\omega)=\frac{\left|S_{y x}(\omega)\right|^{2}}{S_{y}(\omega) \cdot S_{x}(\omega)}
$$

In all what follows the dotted lines are the spectral estimation of the real system and the solid line is the Bode diagram of the linearized model. Two frequency responses are made for the railHPV model validation at two different pressures : $P_{r}=250[$ bar $]$ (figure 9) and $P_{r}=650$ [bar $]$ (figure 10), and the same engine speed : $800[\mathrm{rpm}]$. On figure $9, K_{\text {ehpv }}$ have been tuned to 35 to have
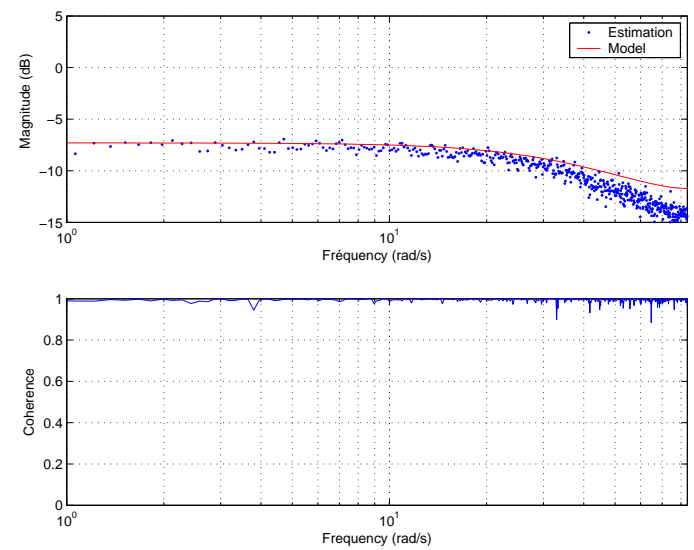

Fig. 9. Rail HPV validation $\left(P_{r}=250\right)$

the same static gain as the one given by the spectral estimation of real data. Keeping the previous
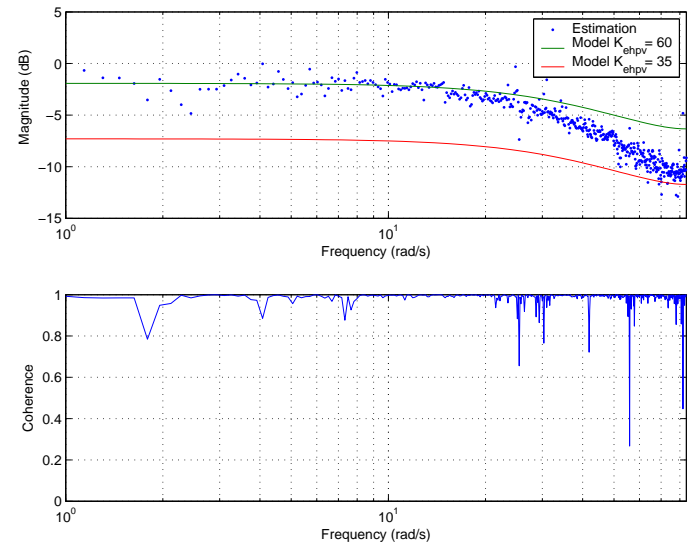

Fig. 10. Rail HPV validation $\left(P_{r}=650\right)$

value of $K_{e h p v}=35$ leads to a biais in the static gain estimation of the real system (lower solid line on figure 10). Some results, not presented in this paper, show that the AmeSim model of the rail HPV provides the same result as the mathematical model. Indeed the electromagnetic phenomena are not modelled in Amesim. In fact, several tests on mathematical model and AmeSim model show that the frequency response does not change significantly according to the operating point (i.e. different positions of the poppet valve, pressures, HPV flow, etc...). We therefore focus on the value of $K_{e h p v}$ which is the electromagnetic gain. Increasing $K_{\text {ehpv }}$ up to 60 gives a better result (upper solid line on figure 10) but the high frequencies remain insufficiently filtered. Several tests on the real system provide a variation of the static gain between $0 d B$ and $-15 d B$, and a variation of high frequencies filtration close to 

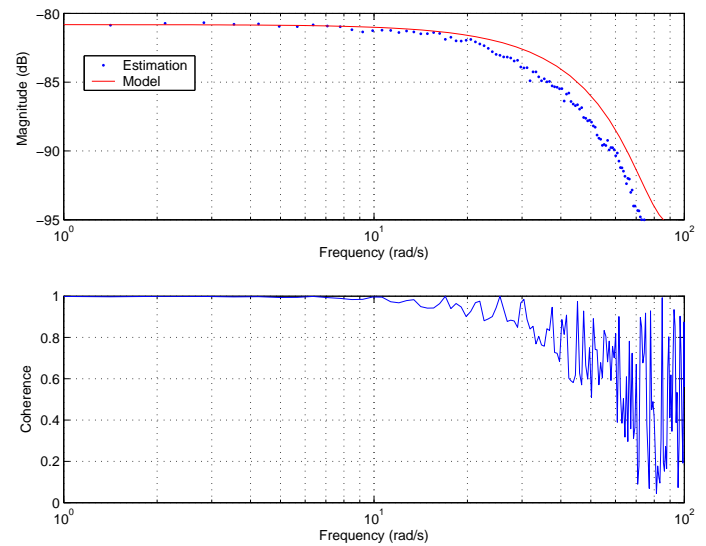

Fig. 11. Frequency response of the HP pump

the ones seen in figure 9 and 10. Concerning now the validation of the model of the HP pump, a comparison between the mathematical model (solid line) and the AmeSim one (dotted line) is presented in Figure 11. The result is considered

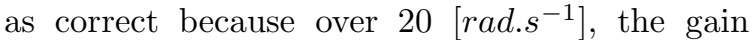
error appears simultaneously with the coherence function decrease.

\section{CONCLUSION}

A physical model of a Diesel Engine Common Rail Injection System has been developed and validated through time and frequency responses. The validated simulator will then be used to evaluate different control schemes, thus limiting the number of real experiments. Moreover, its open structure allows for testing different system configurations, e.g. modification of the HP pump or of the number of injectors. This will avoid carrying out several a posteriori real experiments and therefore reduce the synthesis time of the rail pressure controller. The next step, for control purpose, concerns the system identification over all operating points, as well as the performance specifications.

\section{REFERENCES}

Chebassier, J. (2001). Methodology for the design of a computer control system (in french). PhD thesis. Laboratoire d'Automatique de Grenoble, INPG.

Gissinger, G. and N. Le Fort-Piat (2002). Contrôle-commande de la voiture. Lavoisier.

Guerrassi, N. and P. Dupraz (1998). A common rail injection system for high speed direct injection diesel engines. International Congress Exposition : Diesel Fuel Injection Sprays, SAE no 980803.

Guerrassi, N., D. Schoeppe, Ph. Bercher and P. Spadafora (2002). Common rail injection system design and strategies for low emissions diesel vehicles. The 2nd International Conference on Advanced Automotive Diesel Engine Technology, SAE-China pp. 125, 133.

IMAGINE (2003). AmeSim, User manual and Software.

Kiencke, U. and L. Nielsen (2000). Automotive Control Systems. Springer.

Ljung, L. (2001). System Identification, theory for the user, Second Edition. Prentice hall.

Rucker, J., F. Richert, A. Schlober, D. Abel, O. Hermann, S. Pischinger and A. Pfeifer (n.d.). A model based predictive attempt to control boost pressure and egr-rate in a heavy duty diesel engine.

van Nieuwstadt, M., P. Moraal and I. Kolmanovsky (1999). Optimal control of variable geometry turbocharged diesel engines with exhaust gas recirculation. Vol. 67. Proc of ASME Dynamic Systems and Control Division. pp. 265, 273. 